\title{
Suction Break During Femtosecond Laser-Assisted Cataract Surgery and Misplaced Laser Beam Delivery to the Corneal Layers
}

This article was published in the following Dove Press journal: International Medical Case Reports Journal

\author{
Takeshi Teshigawara $\mathbb{D}^{1-3}$ \\ Akira Meguro iD ${ }^{3}$ \\ Nobuhisa Mizuki ${ }^{3}$ \\ 'Yokosuka Chuoh Eye Clinic, Yokosuka, \\ Kanagawa, Japan; ${ }^{2}$ Tsurumi Chuoh Eye \\ Clinic, Yokohama, Tsurumi, Japan; \\ ${ }^{3}$ Department of Ophthalmology, \\ Yokohama City University School of \\ Medicine, Yokohama, Kanazawa, Japan
}

Correspondence: Takeshi Teshigawara Yokosuka Chuoh Eye Clinic, 2-6 Odakicho, Yokosuka, Kanagawa 238-0008, Japan Tel +8I 46827400 I

Fax +8I 46827414 I

Email teshitake@gmail.com

\begin{abstract}
A 68-year-old man with senile cataract underwent femtosecond laser-assisted cataract surgery (FLACS) in his left eye. Only anterior capsulotomy and lens fragmentation were planned with a femtosecond laser. Docking of the patient interface and anterior capsulotomy were completed without any complications. During the lens fragmentation process, the patient could not resist the temptation to squeeze his eyes shut, which caused excessive pressure from the eyelids. As the procedure proceeded, a bubble was formed at the edge of the patient interface and became increasingly larger. In addition, wrinkles in the conjunctiva were observed. As the lens fragmentation was approaching the final stage, the surgeon was reluctant to release the foot pedal to stop the laser emission. Finally, the patient interface lost adhesion to the cornea. Owing to the high repetition rate of the laser, the laser beam slipped into the corneal layers Under an operating microscope, a grid-pattern laser beam trace was observed in the peripheral part of the cornea. As posterior capsule rupture occurred during the lens removal process, IOL insertion was no longer a suitable option. Therefore, scleral fixation of the implanted intraocular lens was performed without any unexpected events. One year postoperatively, the laser beam trace in the corneal layers could still be identified by slit-lamp examination. Nonetheless, since the laser beam trace was limited to the peripheral part of the cornea, and there was no damage to the central cornea, the visual acuity was 20/20. FLACS has significant benefits, especially in challenging cases of cataract surgery, and has well-established built-in safeguards for complications. However, this case study indicates the possibility of a suction break during laser emission and the preoperative risk factors. It demonstrates that recognizing the signs of suction break is necessary to avoid misplacement of the laser beam on the corneal layers.
\end{abstract}

Keywords: FLACS, complication, safeguard, laser beam trace

\section{Introduction}

High-precision femtosecond laser-assisted cataract surgery (FLACS) was reported in an initial clinical study by Nagy et al in 2009. ${ }^{1}$ FLACS consists of corneal incision, capsulotomy, and lens fragmentation. This technology provides various advantages for cataract surgery, such as reduction in ultrasound energy, increased capsulotomy precision, and decreased postoperative corneal endothelial loss and inflammation. ${ }^{2}$ Similar to other new technologies, FLACS has a learning curve, and some complications related to FLACS have 


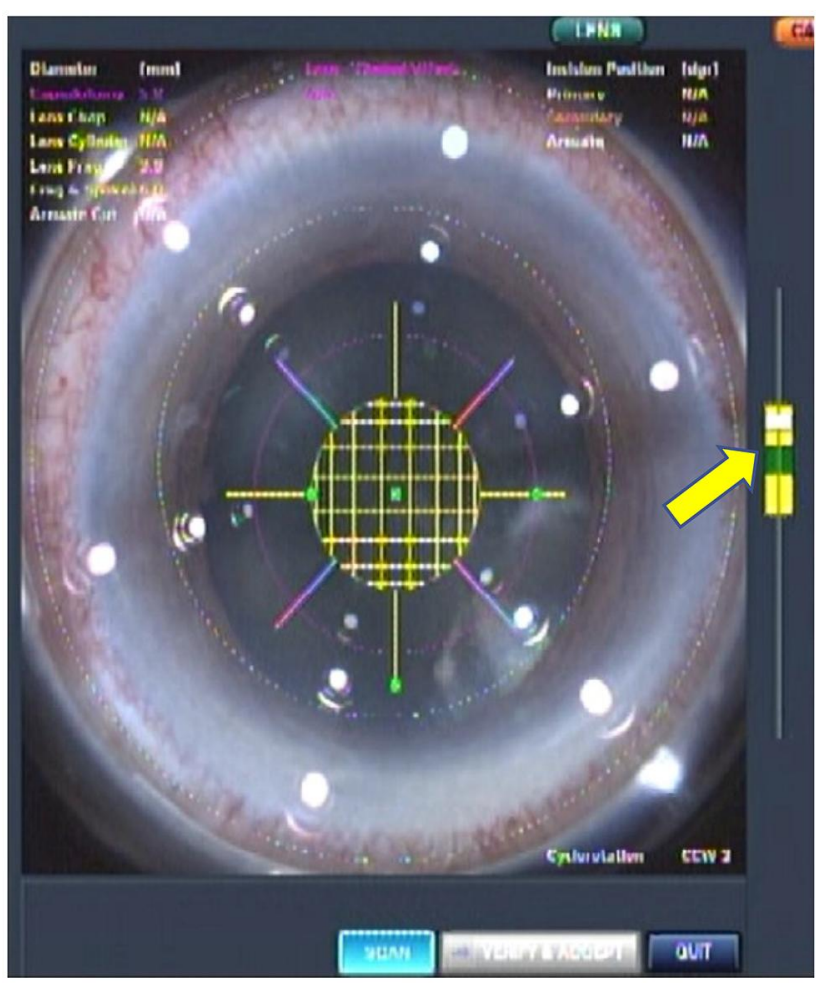

Figure I View of the patient interface. The vacuum-level gauge is within normal limits (yellow arrow).

also been reported, including suction break during laser beam emission. ${ }^{3}$ While the rate of suction break reported by different researchers was low, ${ }^{4}$ some reports indicated that it could cause tissue damage due to erroneous laser beam emission. ${ }^{5,6}$ However, the safeguards for suction break that are built into FLACS instruments are rarely discussed.

We encountered a rare case in which suction break slowly occurred during lens fragmentation, and the gridpattern laser beam slipped onto the corneal layers. Unlike previous reports related to suction break, this case occurred relatively slowly. Therefore, we could identify the possible indications for the suction break. It may be useful to be familiar with the indications to avoid undesirable tissue damage during FLACS. In this report, in addition to describing our case, the possible signs of suction break and possible future development of laser systems are also discussed.

\section{Case Report}

Detailed information about this case report was provided to the patient before he provided informed consent to publish this case report. This case study was approved by the Ethics Committee of the Yokosuka Chuoh Eye Clinic.

A 68-year-old man was scheduled to undergo FLACS in his left eye. The cataract was classified as grade 3 according to the Emery Little classification. ${ }^{7}$ The corrected distance visual acuity was $20 / 30$ in the left eye. Anterior keratometry values (K1 flat and K2 steep) were 43.75 and 44.00 diopters, respectively. While our patient had diabetes, there was no sign of diabetes-related eye problems. In addition, no other eye problems were observed. There was no sign in his facial structure of possible difficulties in docking between the eye and a new SoftFit patient interface (PI) (Alcon Laboratories, Fort Worth, TX, USA). Therefore, the patient seemed to be a good candidate for FLACS. Only capsulotomy and lens fragmentation were planned with a LenSx ${ }^{\circledR}$ Laser (Alcon Laboratories), followed by normal manual procedures. The patient remained in a flat and stable position. There was no irregularity with the docking process. We confirmed that the patient's eye was correctly aligned to the plane of the PI and was properly centered.

Neither bubbles nor loose conjunctiva was trapped between the eye and PI. The vacuum-level gauge was within normal limits (Figure 1), and an image obtained using built-in anterior segment optical coherence tomography showed no abnormality. The variables for the capsulotomy and lens fragmentation were as follows: for the capsulotomy, a diameter of $5.0 \mathrm{~mm}$, incision depth of $600 \mu \mathrm{m}$, and pulse energy of $5 \mu \mathrm{J}$; for lens fragmentation, 8 chops and $350 \mu \mathrm{m}$ grid pattern, and 10 $\mu \mathrm{J}$ pulse energy. The treatment and safety zones were set appropriately by the operator. Soon after the operator started firing the laser, it was found that a small bubble appeared at 3 o'clock and disappeared (Figure 2). In hindsight, this might have been an initial sign of insufficient suction.

During capsulotomy, the small bubble disappeared. No remarkable problem was noted, and a capsulorhexis was created. In the second half of the lens fragmentation process, the small bubble reappeared in the same area immediately after laser beam emission started (Figure 3). Although the operator noticed this, he hesitated to release his foot from the foot pedal to stop the laser beam emission since the process was almost complete. With time, the bubble slowly became bigger and expanded to the central part between the eye and PI. During that time, wrinkles in the conjunctiva and 


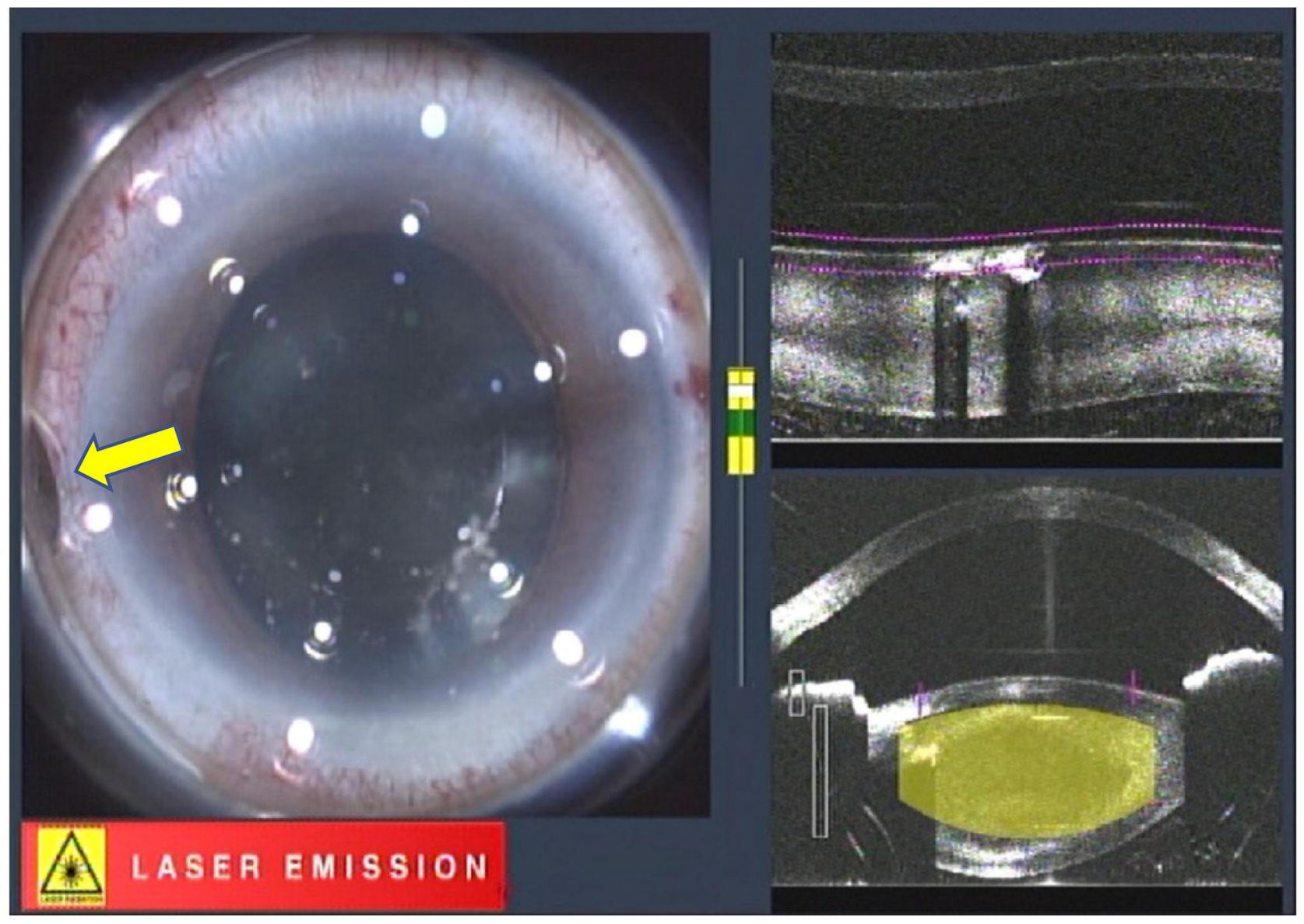

Figure 2 A small bubble is seen appearing at 3'o clock in view of the patient interface (yellow arrow), when laser irradiation began.

a small tilt of the eye were also observed, while the vacuum-level gauge was still within normal limits (Figure 4). Finally, the PI lost adhesion to the sclera (ie, suction break occurred); however, due to the high repetition rate of the laser system, a fraction of a second after the suction break was sufficient for the laser beam to slip onto the corneal layers (Figure 5). Although the operator kept the foot pedal pressed even after the PI was detached from the eye, an automatic laser emission-stop system was activated (Figure 6).

A corneal incision was manually created as planned. The completion of the capsulotomy was confirmed, and the free-floating capsule was removed with capsule forceps. During removal of the fragmented lens with the laser, a grid-pattern laser beam trace was observed between 2 o'clock and 4 o'clock, between the corneal apex and corneal limbus (Figure 7). Since the posterior capsule rupture occurred during the lens removal process, which was unrelated to the suction break, scleral fixation of intraocular lens implantation was performed without unexpected events.

On the day of the operation, a Descemet fold and swelling of the corneal stroma were found in the same area, where the laser beam had been accidentally misplaced. Two months postoperatively, the Descemet fold and swelling of the corneal stroma completely disappeared. However, the grid-pattern laser beam trace was still observed in the cornea (Figure 8). One year postoperatively, the overall visual function was satisfactory to the patient. The corrected distance visual acuity was 20/20. Neither corneal scarring nor opacity was observed. Endothelial cell density was 2512 cells $/ \mathrm{mm}$, as checked using a CellChek XL (Konan Medical, Hyogo, Japan). The contrast sensitivity, tested using a Takagi Glare Tester CGT-1000 (Takagi Seiko, Nagano, Japan), was within normal limits. There were 


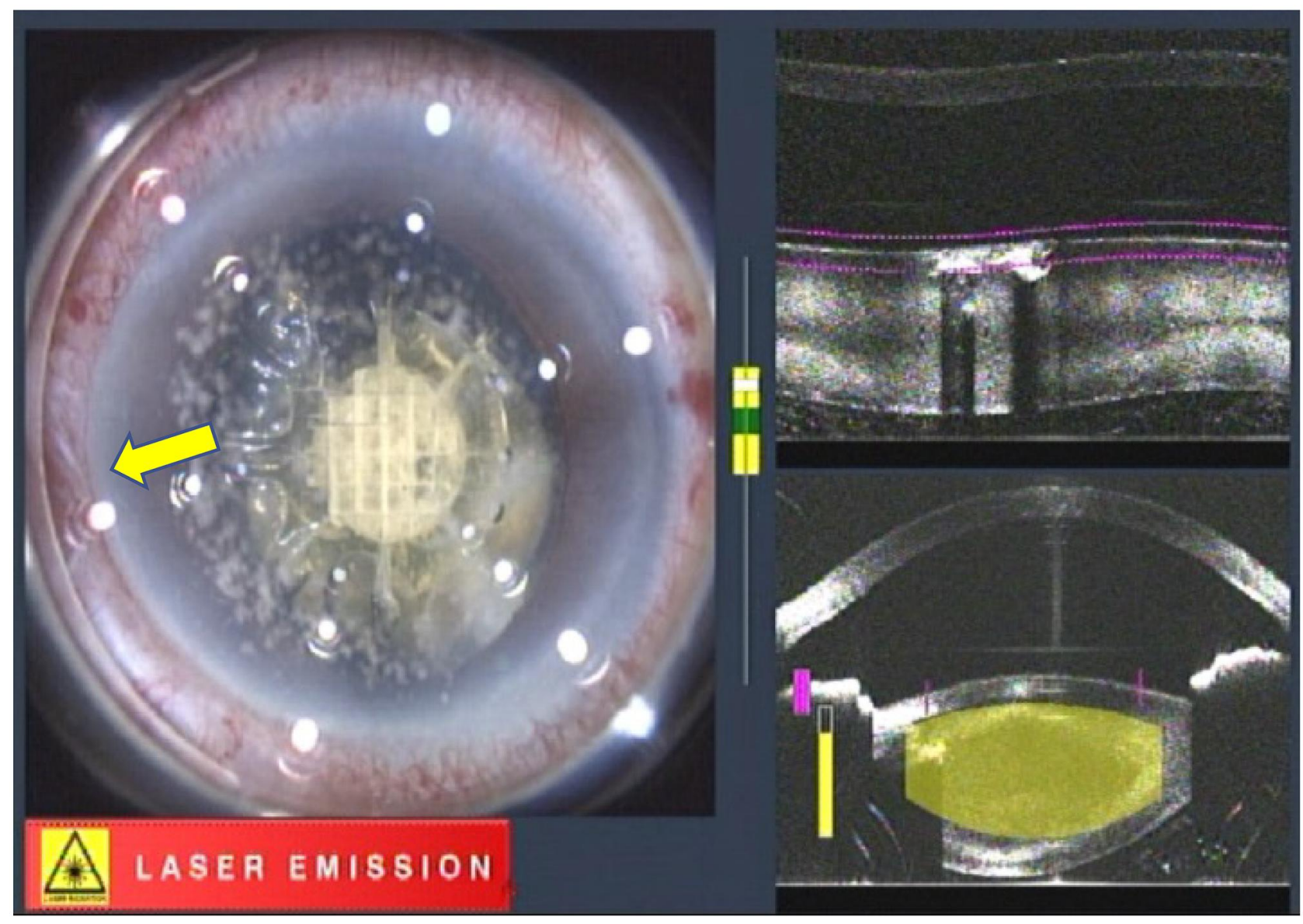

Figure 3 During lens fragmentation, the bubble is still observed (yellow arrow).

no symptoms of photic phenomena, such as a halo or glare.

\section{Discussion}

The advent of FLACS has provided cataract patients and surgeons with several benefits, especially in challenging cases. ${ }^{8-12}$ Crema et al reported FLACS to be effective in patients with weak zonular and even lens subluxation and useful in preventing further zonular damage. ${ }^{8}$ Vasavada et al concluded that compared to conventional phacoemulsification, FLACS showed a lesser decrease in endothelial cell density, less inflammation, and better vision in the early postoperative period in patients with a shallow anterior chamber. ${ }^{9}$ Additionally, compared with conventional phacoemulsification, FLACS has an advantage in managing hard nuclear and white cataracts. $^{10,11}$ Furthermore, in unusual cataracts, such as posterior polar cataracts, FLACS has shown enhanced safety. ${ }^{12}$
Thus, FLACS has many safety advantages during cataract surgery compared to conventional phacoemulsification. However, like other new surgical techniques, FLACS has a steep learning curve with some complications, which surgeons should be aware of. Nagy et al reported the following complications related to FLACS: suction break, conjunctival redness or hemorrhage, capsule tags and bridges, anterior tear, miosis, and endothelial damage due to cutting within the endothelial layer. ${ }^{3}$

They indicated that the probability of a suction break was $2 \%$ before the PI was improved. However, this did not occur after the PI was improved. ${ }^{3}$ Therefore, today, cases of suction break are very rare, and even if suction break occurs, the corneal incision, capsulotomy, and lens fragmentation processes can be completed manually. Nonetheless, a few case studies of suction breaks reported that the laser beam was accidentally applied to the corneal layer due to the high laser repetition rate. ${ }^{5,6}$ Therefore, users still need to be familiar with 


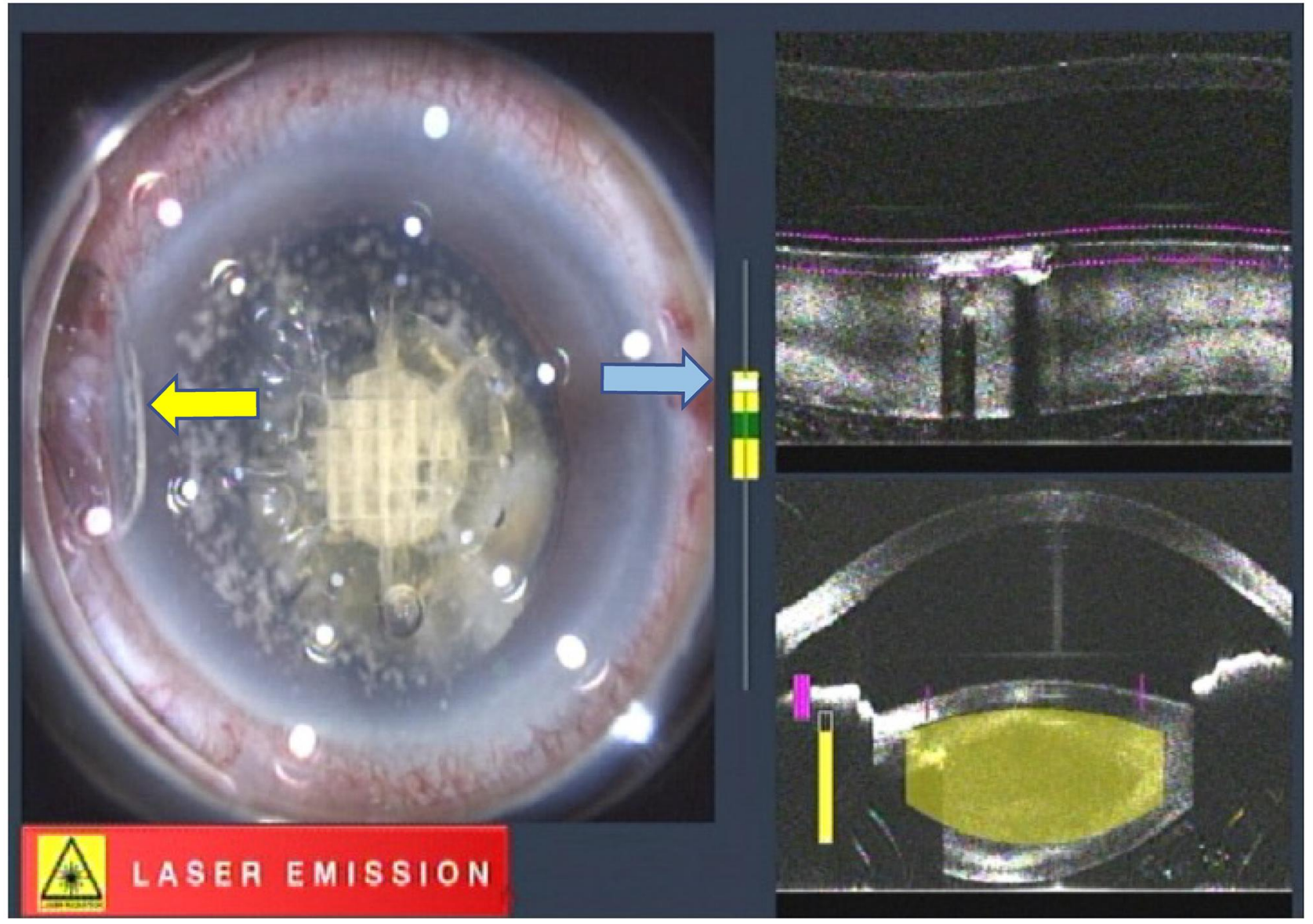

Figure 4 The bubble slowly becomes bigger. Wrinkles in the conjunctiva (yellow arrow) and a small eye tilt are also observed. The vacuum-level gauge is still within normal limits (blue arrow).

possible precautions and consequences of a suction break.

There are some risk factors for a suction break. ${ }^{13}$ Pterygium, narrow lid margin, loose conjunctiva, too flat or steep cornea, and chemotic conjunctiva are common preoperative risk factors. During the operation, a decentered and uneven interface, abrupt eye movement, and excessive pressing of eyelids are common risk factors. In our case, the patient had no preoperative risk factors for a suction break. In this study, the eye docked with the laser device smoothly and was safely adhered to. Unfortunately, the patient's inability to focus on a bright light during laser beam emission caused excessive pressing of the eyelid, and as a result, a suction break occurred. Our experience and that of others indicated that even if precautions are taken, there is still a possibility for a suction break. Therefore, it is crucial to be aware of the indications of suction loss during laser beam emission. Previous reports recorded a sudden suction break, but in our case, a few warning signs appeared before the suction break finally occurred. The following are indications that may be seen before a suction break: 1) fluctuations on the vacuum-level gauge, 2) slow eye tilt, 3) formation of conjunctival wrinkles and 4) formation of bubbles in the peripheral area between the eye and PI, which then increase in size and move toward the central area.

Again, in previous reports, the suction break occurred suddenly when patients moved their faces. In such cases, it would be challenging to detect this sign. However, when suction loss occurs slowly, the signs mentioned earlier may encourage operators to release the foot pedal before delivering the laser beam to the cornea. Unfortunately, we were not familiar with these indications and missed the chance to release the foot 


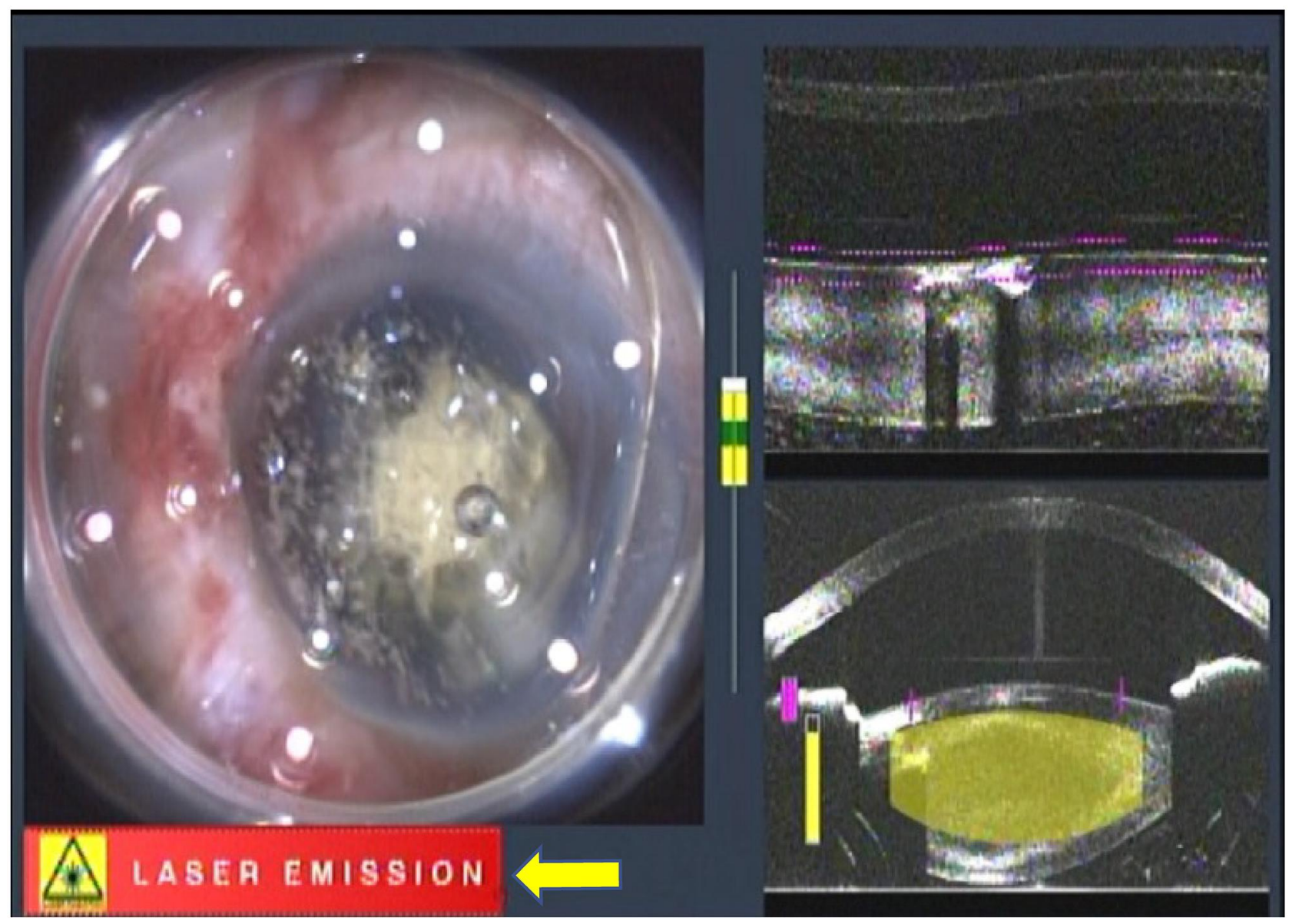

Figure 5 Misplacement of the laser beam in the corneal layers immediately after the suction break. The device is still emitting the laser beam (yellow arrow).

pedal, which caused the grid laser patch to be applied to the corneal layers.

In addition to raising awareness on these issues, more advanced laser safety systems are in development. Currently, available laser safety systems are already equipped with automatic stop systems. However, these systems are activated immediately after the suction break. In other case reports and ours, the high repetition rate of the laser can result in misplacement of the laser to surrounding tissues. Therefore, a faster processor to detect a proximate suction break may be required. Moreover, the current PI cannot detect occlusion of the loose conjunctiva into a suction hole. This phenomenon may affect the accuracy of the vacuum-level gauge. ${ }^{13}$ Furthermore, a better PI is also expected.

In addition, nitrous oxide, the most commonly used inhalation anesthetic in dentistry that provides minimal and moderate sedation, can be a good option to minimize abrupt patient movements. Deep sedation is not a viable option since patients still have to be responsive and focus on lights during the operation. ${ }^{5,12}$ Minimal and moderate sedation with nitrous oxide maintains patient cooperation and even decreases eye movement. ${ }^{14}$ Since nitrous oxide inhalation was introduced as an anesthetic for FLACS in our facility, we have found that this anesthetic is very effective, especially for nervous patients and those who have difficulty keeping their eyes still during the procedures. Moreover, proper patient education can also be useful precautionary measures, such as "Dos and Don'ts instructions" before the laser procedure.

Finally, our report is in agreement with previous reports $^{5,6}$ indicating that a suction break might not be a vision-threatening complication. Nevertheless, like other new technologies, unexpected events may occur. Therefore, preoperative informed consent regarding 


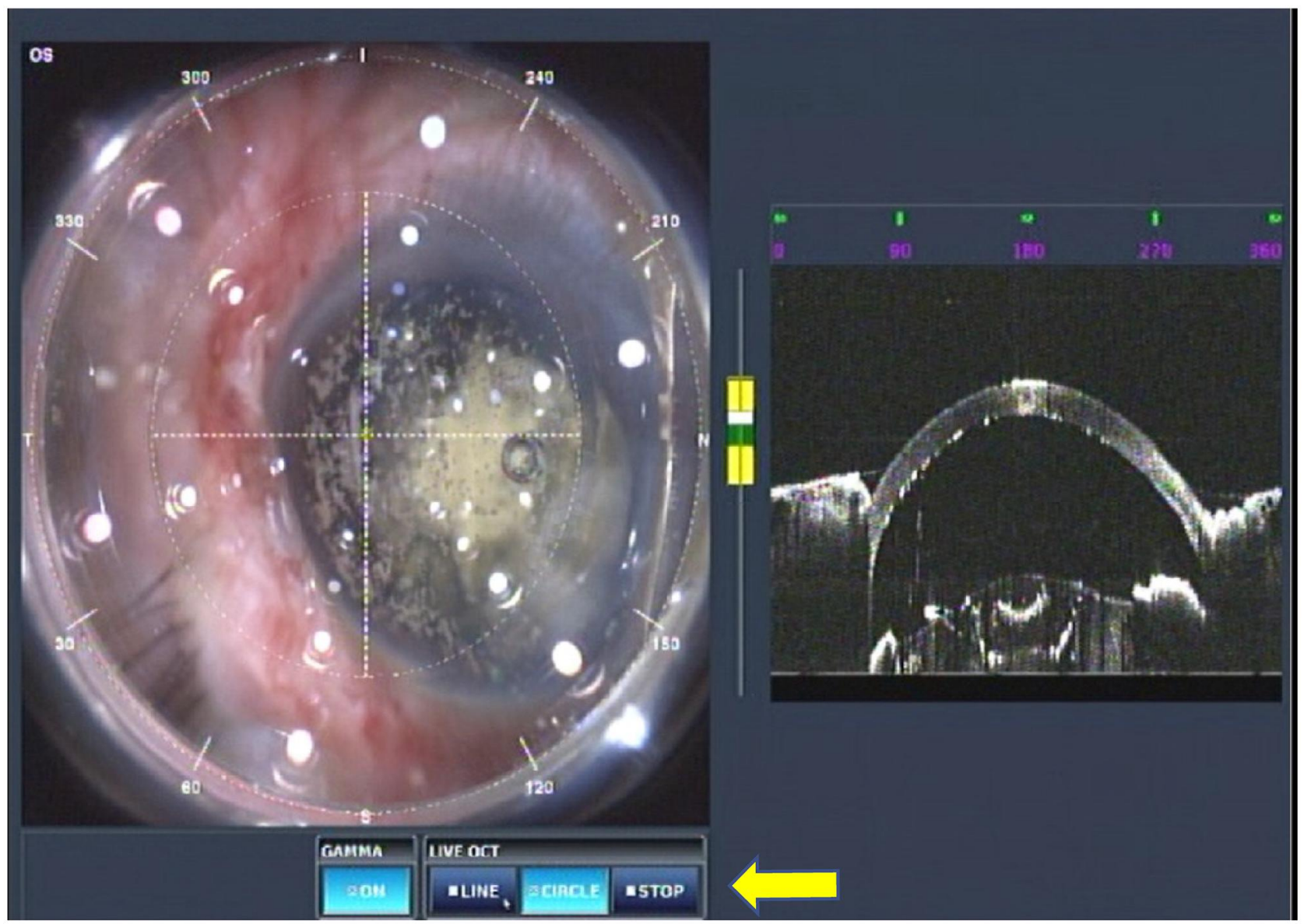

Figure 6 The automatic laser-emission-stop system is activated (yellow arrow).

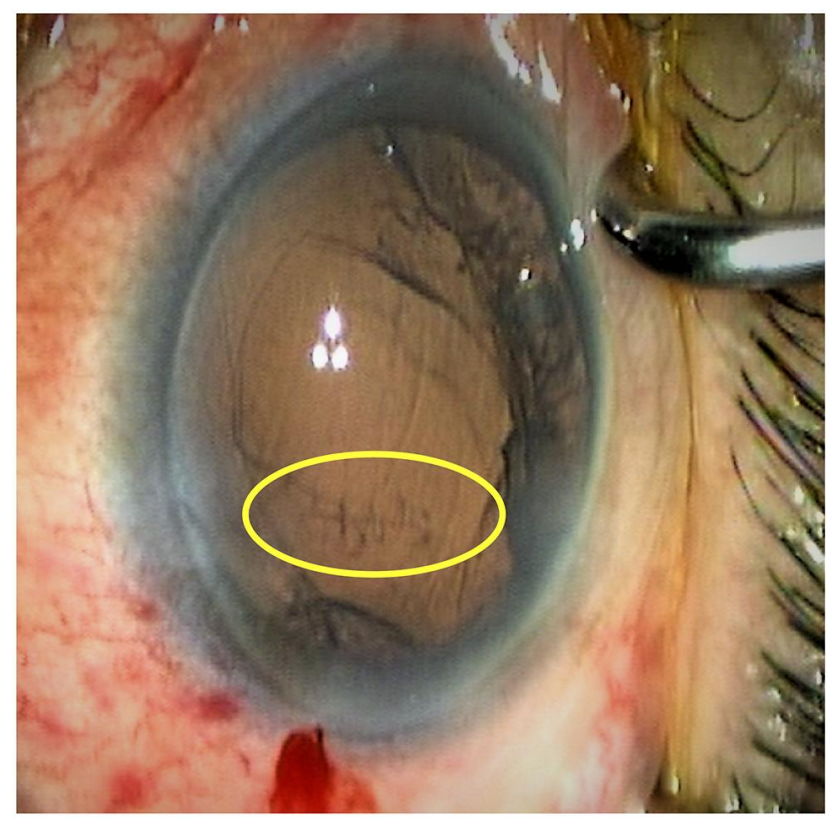

Figure 7 Intraoperative view of the grid-pattern laser beam trace in the cornea layers (yellow circle).

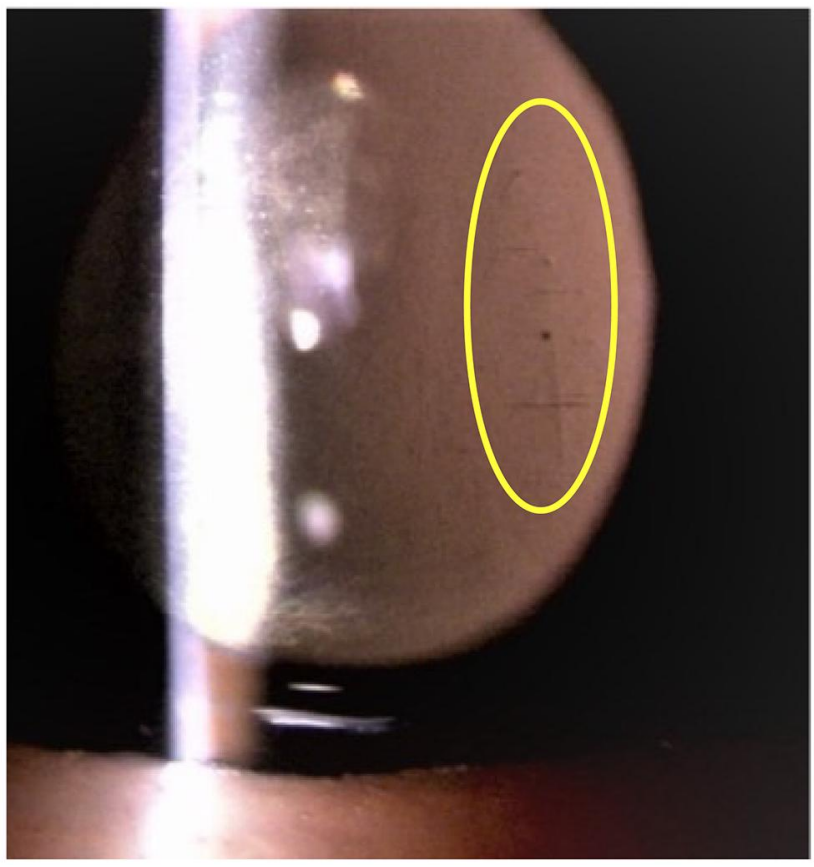

Figure 8 Postoperative slit-lamp image of the grid-pattern laser beam trace I year after surgery (yellow circle). 
complications may be useful when dealing with possible misadventures.

\section{Acknowledgments}

We would like to thank Editage for English language editing.

\section{Disclosure}

The authors report no conflicts of interest in this work.

\section{References}

1. Nagy Z, Takacs A, Filkorn T, Sarayba M. Initial clinical evaluation of an intraocular femtosecond laser in cataract surgery. J Refract Surg. 2009;25(12):1053-1060. doi:10.3928/1081597X-20091117-04

2. Nagy ZZ, Mastropasqua L, Knorz MC. The use of femtosecond lasers in cataract surgery: review of the published results with the LenSx system. $J$ Refract Surg. 2014;30(11):730-740. doi:10.3928/1081597X20141021-04

3. Nagy ZZ, Takacs AI, Filkorn T, et al. Complications of femtosecond laser-assisted cataract surgery. $J$ Cataract Refract Surg. 2014;40 (1):20-28. doi:10.1016/j.jcrs.2013.08.046

4. Ang RET, Quinto MMS, Cruz EM, Rivera MCR, Martinez GHA. Comparison of clinical outcomes between femtosecond laser-assisted versus conventional phacoemulsification. Eye Vis (Lond). 2018;5:8. doi:10.1186/s40662-018-0102-5

5. Kent C. Femto laser cataract: avoiding complications. Rev Ophthalmol. 2014. Available from: https://www.reviewofophthalmology.com/article/ femto-laser-cataract-avoiding-complications. Accessed April 13, 2020.
6. Schultz T, Dick HB. Suction loss during femtosecond laser-assisted cataract surgery. J Cataract Refract Surg. 2014;40(3):493-495. doi:10.1016/j.jcrs.2013.12.004

7. Emery JM. Surgical techniques, complications and results. In: Emery JM, Little JH, editors Phacoemulsification and Aspiration of Cataract. St Louis, MO, USA: CV Mosby; 1979:45-48.

8. Crema AS, Walsh A, Yamane IS, Ventura BV, Santhiago MR. Femtosecond laser-assisted cataract surgery in patients with marfan syndrome and subluxated lens. J Refract Surg. 2015;31(5):338-341. doi:10.3928/1081597X-20150424-02

9. Vasavada VA, Vasavada S, Vasavada AR, Vasavada V, Srivastava S. Comparative evaluation of femtosecond laser-assisted cataract surgery and conventional phacoemulsification in eyes with a shallow anterior chamber. $J$ Cataract Refract Surg. 2019;45(5):547-552.

10. Titiyal JS, Kaur M, Singh A, Arora T, Sharma N. Comparative evaluation of femtosecond laser-assisted cataract surgery and conventional phacoemulsification in white cataract. Clin Ophthalmol. 2016;10:1357-1364. doi:10.2147/OPTH.S108243

11. Chen X, Yu Y, Song X, Zhu Y, Wang W, Yao K. Clinical outcomes of femtosecond laser-assisted cataract surgery versus conventional phacoemulsification surgery for hard nuclear cataracts. Cataract Refract Surg. 2017;43(4):486-491. doi:10.1016/j.jcrs.2017.01.010

12. Vasavada AR, Vasavada V, Vasavada S, Srivastava S, Vasavada V, Raj S. Femtodelineation to enhance safety in posterior polar cataracts. J Cataract Refract Surg. 2015;41(4):702-707. doi:10.10 $16 /$ j.jcrs.2015.02.021

13. Yoo S. How patient movement can interfere with femto laser-assisted cataract surgery. Ophthalmology Times. 2016. Available from: https://www.ophthalmologytimes.com/view/how-patient-movementcan-interfere-femto-laser-assisted-cataract-surgery. Accessed July 5, 2020.

14. Nair G, Kim M, Nagaoka T, et al. Effects of common anesthetics on eye movement and electroretinogram. Doc Ophthalmol. 2011;122 (3):163-176. doi:10.1007/s10633-011-9271-4

\section{Publish your work in this journal}

The International Medical Case Reports Journal is an international, peer-reviewed open-access journal publishing original case reports from all medical specialties. Previously unpublished medical posters are also accepted relating to any area of clinical or preclinical science. Submissions should not normally exceed 2,000 words or 4 published pages including figures, diagrams and references. The manuscript management system is completely online and includes a very quick and fair peer-review system, which is all easy to use. Visit http://www.dovepress.com/testimonials.php to read real quotes from published authors. 